\title{
A Multicomponent Synthesis of Stereodefined Olefins via Nickel Catalysis and Single Electron/Triplet Energy Transfer
}

\author{
Chen $\mathrm{Zhu}^{1,2 \dagger}$, Huifeng Yue $^{1,2 \dagger}$, Bholanath Maity ${ }^{1}$, Iuliana Atodiresei ${ }^{2}$, Luigi Cavallo ${ }^{1 *} \&$ Magnus Rueping ${ }^{1,2^{*}}$
}

Unsaturated carbon-carbon bonds are one of the most common and important structural motifs in many organic molecules, stimulating the continuous development of general, efficient, and practical strategies for their functionalisation. Herein, we report a one-pot difunctionalisation of alkynes via a photoredox/nickel dual catalysed three-component cross coupling reaction under mild conditions, providing access to a series of highly important trisubstituted alkenes. Notably, in contrast to the traditional methods which are based on the steric hindrance of the substrates to control the reaction selectivity, both E- and Z- isomers of trisubstituted alkenes which are often energetically close, can be obtained via choosing an appropriate photocatalyst with a suitable triplet state energy. Beyond the immediate practicality of this transformation, this newly developed methodology might inspire the development of diverse and important one-pot functionalisations of carbon-carbon multiple bonds via photoredox and transition-metal dual catalysed multi-component reactions.

Multi-component reactions are a class of useful transformations employed widely for the efficient synthesis of diverse compounds in organic synthesis ${ }^{1-6}$. In the past decades, significant progress has been achieved in the field of difunctionalisation of carbon-carbon multiple bonds via multi-component reactions. However, the employed methods rely largely on the use of organometallic species such as Grignard reagents ${ }^{7}$, organoboron ${ }^{8}$ and organolithium ${ }^{9}$ reagents and require high temperatures or multistep reactions. More recently, radical-mediated transformations have been developed, providing a good alternative for the one-pot difunctionalisation of these motifs. In this case, a radical is generally generated from a suitable precursor via a single electron transfer process by using $\mathrm{O}_{2}{ }^{10}$, transition metal species ${ }^{11-14}$ or photocatalysts ${ }^{15-17}$. The generated radical adds to the alkyne or alkene moiety to give a vinyl or alkyl radical intermediate. For transition metal catalysed transformations, various radical precursor including sulfonyl chlorides ${ }^{11}$, activated alkyl bromides ${ }^{12}$, and fluoroalkyl iodides ${ }^{13}$ were used in the cross-coupling reactions with organoboron reagents. For photoredox catalysed protocols, in most of the cases, the generated vinyl or alkyl radical intermediate undergoes subsequently hydrogen atom transfer (HAT), $\mathrm{CO}_{2}$ fixation ${ }^{16}$ or another SET process to give a carbocation which can be attacked by a nucleophile ${ }^{17}$ under mild conditions. We questioned whether it would be possible to exploit the vinyl or alkyl radical intermediate, generated via a photoredox catalytic pathway, in transition-metal catalysed cross couplings, thus developing a photoredox and transition-metal dual catalysed multi-component cross coupling platform under mild conditions, transformation which has not been accomplished before. Moreover, the $E / Z$ isomerisation induced by the energy transfer of the generated trisubstituted alkene bearing two similar aryl groups on the same carbon atom may be controlled via a carefully tuned photocatalyst with a suitable triplet energy (Fig. 1b), which is highly challenging if compared to a planar, conjugated $E$ isomer and twisted, deconjugated $Z$ isomer (Fig. 1a) ${ }^{18}$. Recently excellent two-component cross coupling reactions have been achieved in the field of $\mathrm{C}-\mathrm{C}$ and $\mathrm{C}$-heteroatom bond formation via photoredox and nickel dual catalysis ${ }^{19-26}$.

Inspired by these results, we envisaged that a three-component cross coupling reaction involving aryl halides, sodium sulfinates and alkynes is feasible via this strategy, enabling the difunctionalisation of alkynes in a one-pot manner, under mild conditions. However, there are several challenges to be addressed, which mainly include $a$-substituted vinyl sulfones generation via Markovnikov addition of the sulfonyl radical to terminal alkynes ${ }^{23}$, aromatic sulfone formation via cross coupling of sulfinate salts with aryl halides ${ }^{24}$ and control of the Z/E ratio of the generated trisubstituted alkenes. Here we report the aryl-sulfonylation of alkynes with high chemo-, regio-, and stereoselectivity (Fig. 1c). The utility and applicability of this methodology was demonstrated by the high tolerance toward numerous functional groups, the functionalisation of complex molecules which is relevant for the late stages of multistep syntheses, the success of gram-scale reactions, and the transformation of the generated products into further valuable organic

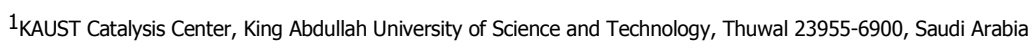

2Institute of Organic Chemistry, RWTH Aachen University, Landoltweg1, 52074 Aachen, Germany

$\dagger$ These authors contributed equally to this work. 
compounds. Our results indicate the reaction outcome is depending on the photocatalyst, that is involved in single electron transfer processes or both, single electron transfer and energy transfer processes.

a

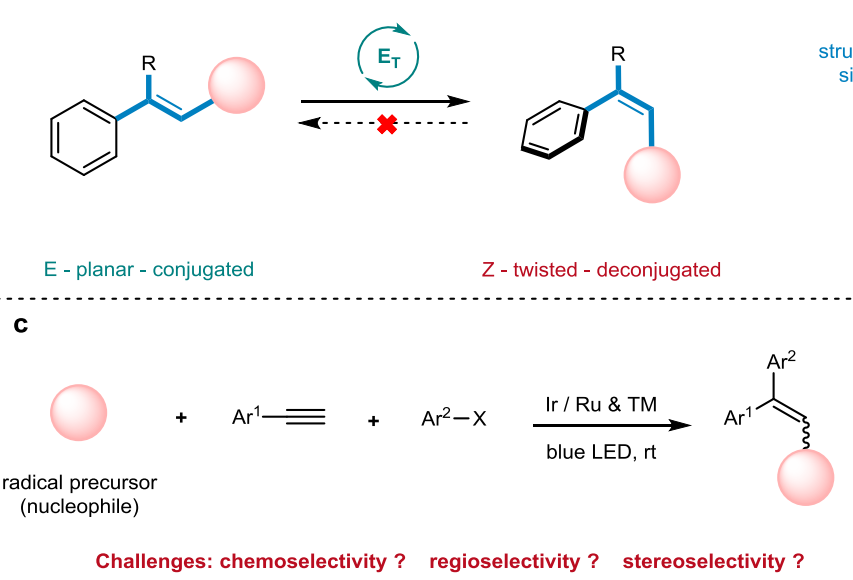

b

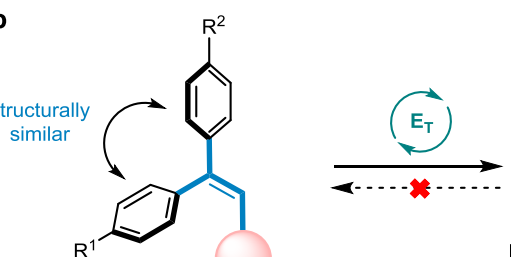

E - twisted - deconjugated

Photo/nickel dual catalyzed three-component cross coupling under mild conditions

$\checkmark$ feedstock aryl halide $\checkmark$ no external base $\checkmark$ room temperature

stereoselective $\quad \downarrow 100$ examples $\checkmark$ scalable

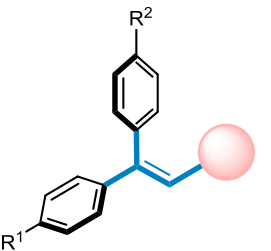

Z - twisted - deconjugated

Figure 1 | Multicomponent cross-coupling reaction under mild conditions and its main challenges. a, Conventional energy transfer induced isomerisation between planar, conjugated $E$ isomer and twisted, deconjugated $Z$ isomer. b. Challenging $E / Z$ isomerisation between two twisted and deconjugated isomers. c, Our proposed multicomponent cross-coupling protocol and its main challenges.

\section{Results}

Mechanistic design. A proposed mechanism for our designed three-component cross coupling platform is shown in Fig. 2. Initially, in the photocatalytic cycle, the $\mathrm{Ir}^{\mathrm{III}}$ or $\mathrm{Ru}^{\mathrm{II}}$ complex absorbs visible light and promotes a long-lived ${ }^{*} \mathrm{Ir}^{\mathrm{III}}$ or ${ }^{*} \mathrm{Ru}^{\mathrm{II}}$ triplet excited state, which then oxidises the radical precursor to the corresponding radical along with formation of the $\operatorname{Ir}^{\mathrm{II}}$ or $\mathrm{Ru}^{\mathrm{I}}$ reductant. The resulting radical enters the nickel catalytic cycle by adding to a $\mathrm{Ni}^{\mathrm{I}}$ complex, which subsequently undergoes reduction, 1,2 migratory insertion of alkyne, Ni-assisted anti/syn isomerisation, oxidative addition of the aryl halide and reductive elimination to give the three-component anti-addition product $\mathbf{P}_{\text {anti }}$. Noteworthy, depending on the photocatalyst, an energy transfer (ET) process promoting the isomerisation of the product can be involved in the transformation. ${ }^{18,27-29}$ The use of photocatalysts with a triplet state energy below that of the excited state of the anti-addition product $\mathbf{P}_{\text {anti }}$, which is $\mathrm{E}_{\mathrm{T}}(\mathbf{P C})<\mathrm{E}_{\mathrm{T}}\left(\mathbf{P}_{\text {anti }}\right)$, results in little or no isomerisation, giving the anti-addition product $\mathbf{P}_{\text {anti }}$ as single product. In contrast, the use of photocatalysts with a triplet state energy higher than that of both isomers, which is $\mathrm{E}_{\mathrm{T}}(\mathbf{P C})>\mathrm{E}_{\mathrm{T}}\left(\mathbf{P}_{\text {syn }}\right)>\mathrm{E}_{\mathrm{T}}\left(\mathbf{P}_{\text {anti }}\right)$, affords the products with low Z/E ratios. However, the use of photocatalysts with a triplet state energy higher than that of the excited state of the anti-addition product $\mathbf{P}_{\text {anti }}$ but lower than that of the corresponding isomeric product $\mathbf{P}_{\text {syn }}$ which is $\mathrm{E}_{\mathrm{T}}\left(\mathbf{P}_{\text {syn }}\right)>\mathrm{E}_{\mathrm{T}}(\mathbf{P C})>\mathrm{E}_{\mathrm{T}}\left(\mathbf{P}_{\text {anti }}\right)$, leads to effective isomerisation, delivering mainly the corresponding isomeric product $\mathbf{P}_{\text {syn }}$ (Fig. 2a). 


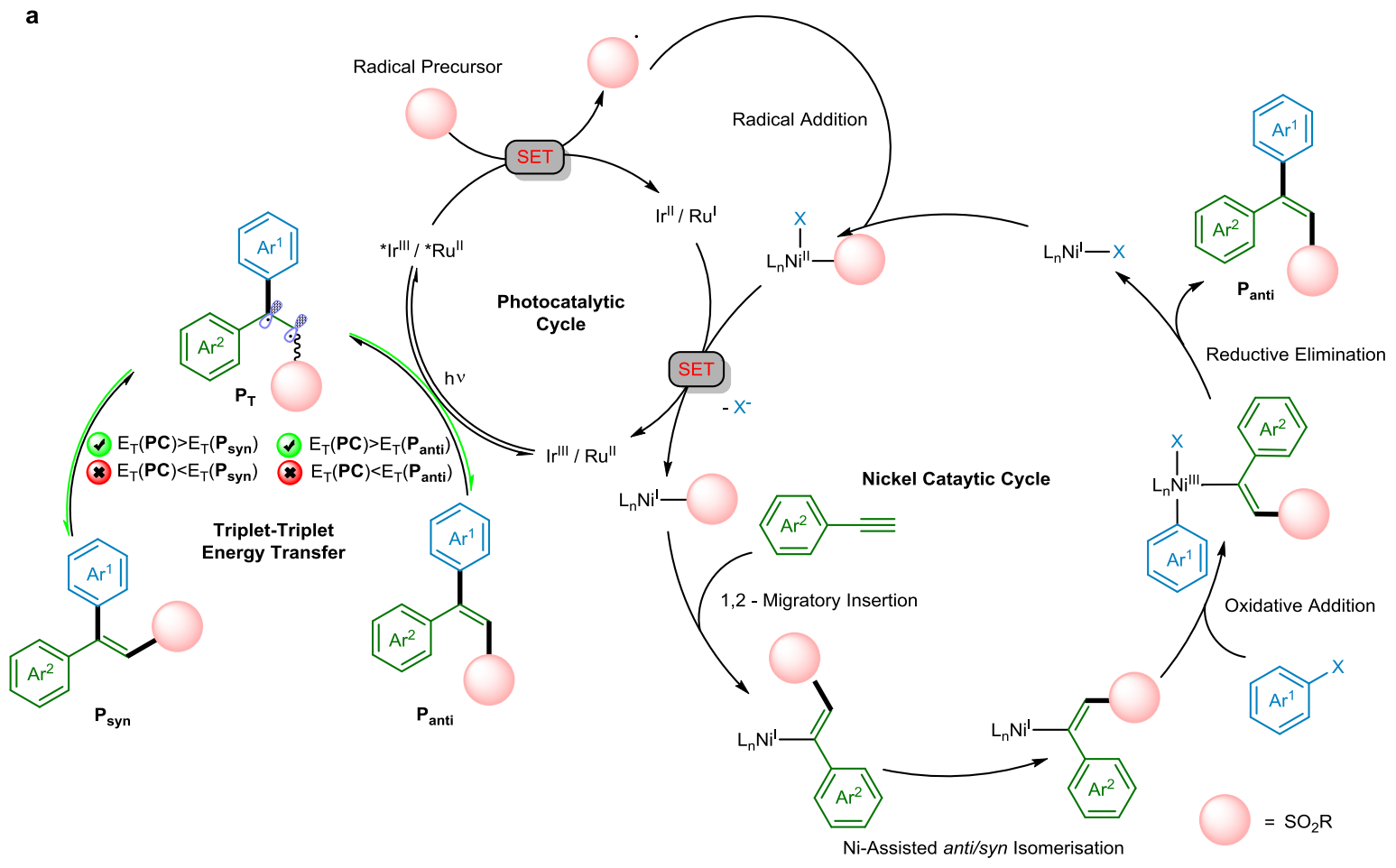

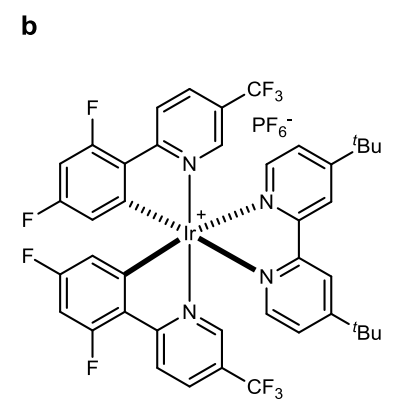

$1,\left(E_{T}=59.4 \mathrm{kcal} / \mathrm{mol}\right)$

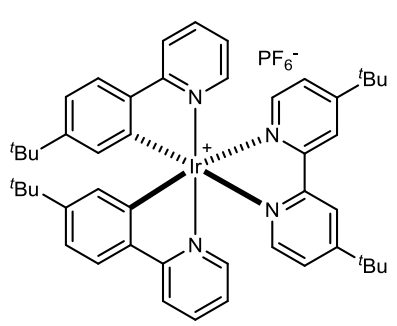

2, $\left(E_{\mathrm{T}}=50.7 \mathrm{kcal} / \mathrm{mol}\right)$<smiles></smiles>

\begin{tabular}{|c|c|}
\hline Pc. & P $_{\text {syn: }}: \mathbf{P}_{\text {anti }}$ \\
\hline Ir $\mathbf{1}$ & $62: 38$ \\
\hline Ir $\mathbf{2}$ & $89: 11$ \\
\hline Ru $\mathbf{3}$ & $1: 99$ \\
\hline
\end{tabular}

3, $\left(\mathrm{E}_{\mathrm{T}}=46.9 \mathrm{kcal} / \mathrm{mol}\right)$

Figure 2 Mechanistic design of the newly developed three-component reaction. a, Excitation of the photocatalyst by visible light to produce a long-lived triplet excited state which oxidises the radical precursor to the corresponding radical (photocatalytic cycle) which enters the nickel catalytic cycle. The resulting $\mathrm{Ni}$-complex undergoes reduction, 1,2-migratory insertion of alkyne, $\mathrm{Ni}$-assisted isomerisation, oxidative addition of aryl bromide and reductive elimination to give the anti-addition three-component product $\mathbf{P}_{\text {anti }}$ (nickel catalytic cycle). In addition, isomerisation can be achieved by triplet energy transfer with the photocatalyst to give the isomeric product $\mathbf{P}_{\text {syn }}$ (triplet-triplet energy transfer). b. Triplet state energy of photocatalysts measured in DMF.

Reaction development. With this design in mind, we started our study by evaluating the cross-coupling reaction between 4-bromobenzonitrile, phenylacetylene and sodium 4-methylbenzenesulfinate in the presence of photocatalyst $2, \mathrm{NiCl}_{2} \cdot \mathrm{dme}$ and dtbbpy ligand (L1) for $12 \mathrm{~h}$. However, only unwanted two-component reaction products such as the corresponding sulfonylated arene $(21 \%)^{24}$ and $\alpha$-substituted vinyl sulfone $(6 \%)^{23}$ as well as 4-hydroxybenzonitrile (20\%) were obtained. Given the essential role of ligands in cross-coupling transformations, we next evaluated several nitrogen and phosphine-containing ligands (see Supplementary Table 1). Notably, 95\% yield of the desired three-component cross coupling product 5 (Z/E ratio 89:11) was obtained when ligand $\mathbf{4}$ was used. Moreover, further investigation of a series of photocatalysts showed that a reverse Z/E ratio could be achieved upon using photocatalysts possessing different triplet state energies (Supplementary Table 4). When photocatalyst $\mathbf{1}$ was used, we obtained the desired product 5 in 49\% yield with 62:38 Z/E ratio. However, when Ru-based photocatalyst 3 was used, the $Z / E$ ratio reached the level of 1:99, emphasizing the unique feature of the developed photoredox and nickel catalysed three-component cross coupling transformation (Fig. 2b). Control experiments demonstrated that no desired product was generated in the absence of light, photocatalyst, nickel catalyst or ligand (Supplementary Table 6).

Scope of the reaction. With the optimised reaction conditions in hand, we first examined the scope of the reaction with respect to the aryl halides using the Ru-based photocatalyst 3. As shown in Fig. 3a, a series of aryl bromides and aryl iodides bearing 
electron-withdrawing, electron-neutral and electron-donating substituents underwent this transformation providing the corresponding products 5-22 in moderate to excellent yields. 2-Methoxyiodobenzene gave a lower yield compared to 4-methoxyiodobenzene, indicating that the reaction was slightly hampered by increasing the steric hindrance (20 and $\mathbf{2 1})$. Notably, substrates bearing common sensitive functional groups such as aldehyde, unprotected aniline and phenol underwent the reaction and gave the corresponding products 11, 23 and $\mathbf{2 4}$ in moderate to high yields, which are hard to achieve using traditional synthetic methods. In addition, this transformation proceeded chemoselectively with aryl iodide over fluoride, chloride and bromide, enabling further functionalisation of the generated products (25-27, 83-99\%). Moreover, disubstituted aryl halides and bicyclic substrates including dioxole, phthalide, phthalimide, and naphthyl derived halides could also be applied in reaction with good to high efficiency $(\mathbf{2 8 - 3 4}, 61-90 \%)$. It is noteworthy that this mild three-component cross coupling method could be readily extended to pharmaceutically relevant heteroaromatic halides. Not only electron-deficient but also electron-rich nitrogen containing heterocyclic halides including quinoline, pyridines, pyrimidine, and pyrazole derivatives could undergo this reaction in good to excellent yields $\mathbf{( 3 5 - 4 1}, 64-95 \%)$. The reaction proceeded smoothly also with thiophene-derived halides, providing the corresponding products $\mathbf{4 2}$ and $\mathbf{4 3}$ in good to high yields. Encouraged by these results, we continued to explore the substrate scope with respect to the structure of alkynes under the standard reaction conditions (Fig. 3b). The generality of alkynes scope was well illustrated by the tolerance of a series of functional groups such as ester, fluoride, chloride, bromide, alkyl, methoxy, methylthio, amine, acetal and boronic ester groups (44-60). Notably, the steric properties of the alkynes have no obvious effects on the reaction efficiency (49-51 and 54-55). Also, 3-ethynylthiophene gave the desired product in good yield (61). More importantly, the scope is not limited to aromatic terminal alkynes, cyclohexenyl alkyne and internal alkynes were also suitable substrates for this catalytic system (62-64). However, the size of the alkyne substituent has a significant effect on the transformation efficiency and the yield decreased as the size of the group increased. 

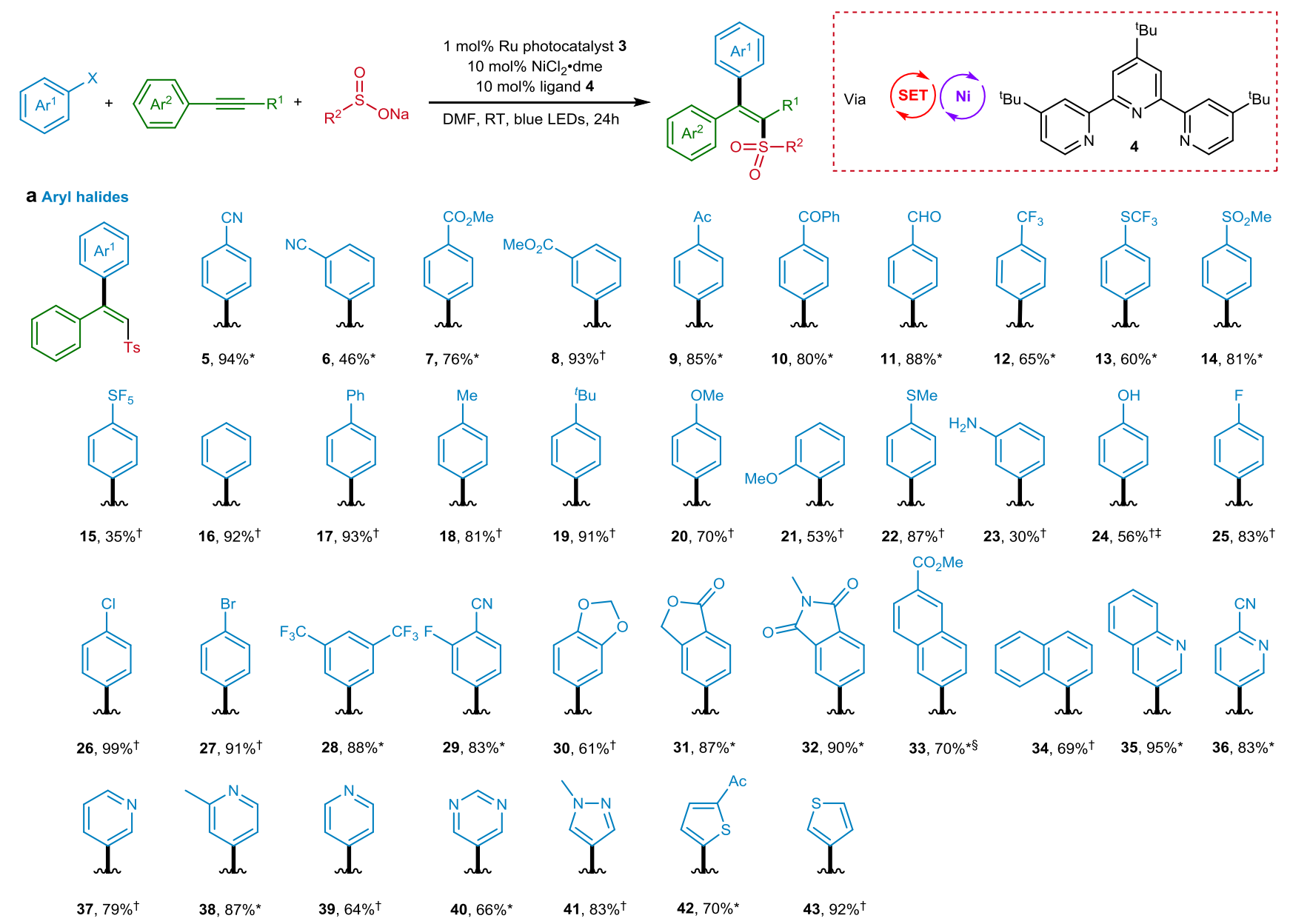

b Alkynes*

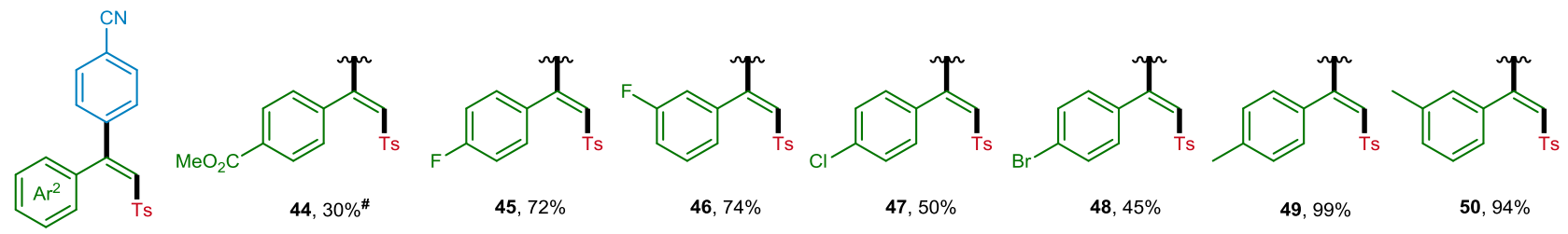

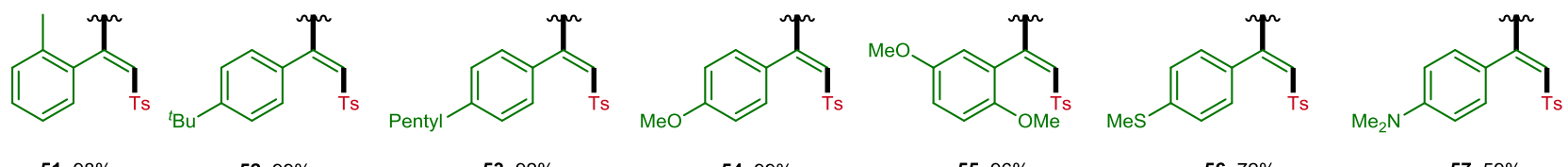

$\begin{array}{llllll}\mathbf{5 1}, 98 \% & \mathbf{5 2}, 99 \% & \mathbf{5 3}, 92 \% & \mathbf{5 4}, 99 \% & \mathbf{5 5}, 96 \% & \mathbf{5 6}, 72 \%\end{array}$<smiles>CC(C)=CCc1ccc(C(C)C)cc1</smiles>

Figure 3 | Substrate scope of aryl halides and alkynes for the photoredox/nickel dual catalysed aryl-sulfonylation of alkynes using Ru photocatalyst 3. Reaction conditions: Aryl halide $(0.4 \mathrm{mmol})$, alkyne $(0.4 \mathrm{mmol})$, sodium 4-methylbenzenesulfinate $(0.2 \mathrm{mmol})$, Ru photocatalyst $3(0.002 \mathrm{mmol}), \mathrm{NiCl} 2 \cdot \mathrm{dme}(0.02$ mmol), ligand $4(0.02 \mathrm{mmol})$, degassed DMF ( $2 \mathrm{~mL})$, room temperature, irradiation with $7.4 \mathrm{~W}$ blue LED strips with fan cooling for $24 \mathrm{~h}$. Yield after purification. *From aryl bromide $\uparrow$ From aryl iodide $\ddagger$ The $Z / E$ ratio of product $\mathbf{2 4}$ is 37:63. § The Z/E ratio of product 33 is 10:90. \# Alkyne as limiting reagent (0.2 mmol), aryl halide $(0.4 \mathrm{mmol})$, sodium sulfinate $(0.4 \mathrm{mmol})$.

Next, we investigated the scope of sodium sulfinates (Fig. 4a). A series of structurally diverse sodium aryl sulfinates bearing electron-donating as well as electron-withdrawing functional groups underwent the reaction smoothly (65-72). While a substrate possessing a methyl group in the ortho position of the aromatic ring worked well $(\mathbf{6 7}, 90 \%)$, a more sterically hindered substrate gave the desired product in moderate yield $(68,53 \%)$. Moreover, sodium naphthyl and thienyl sulfinates were also suitable substrates $(\mathbf{7 3}, 98 \%, \mathbf{7 4}, 97 \%)$. Remarkably, sodium alkyl sulfinates also showed high reactivity in this catalytic system. Sodium 
sulfinates bearing methyl, ethyl, cyclohexyl, and cyclopropyl groups gave the corresponding products 75-78 in 78-92\% yield.

Finally, a series of complex molecules were evaluated in this protocol (Fig. 4b). For example, aryl halides bearing galactopyranose, probenecid, pregnenolone, cholestanol, and adamantanecarboxylic acid motifs reacted with phenylacetylene and sodium 4-methylbenzenesulfinate to give the appropriate products $\mathbf{7 9 - 8 3}$ in moderate to high yields. Estrone and $\delta$-tocopherol derived alkynes underwent reaction with 4-bromobenzonitrile and sodium 4-methylbenzene-sulfinate to give the corresponding products $\mathbf{8 4}$ and 85 with good efficiency. Notably, trisubstituted alkene 86, bearing two complex motifs, was achieved in $84 \%$ yield via this strategy.

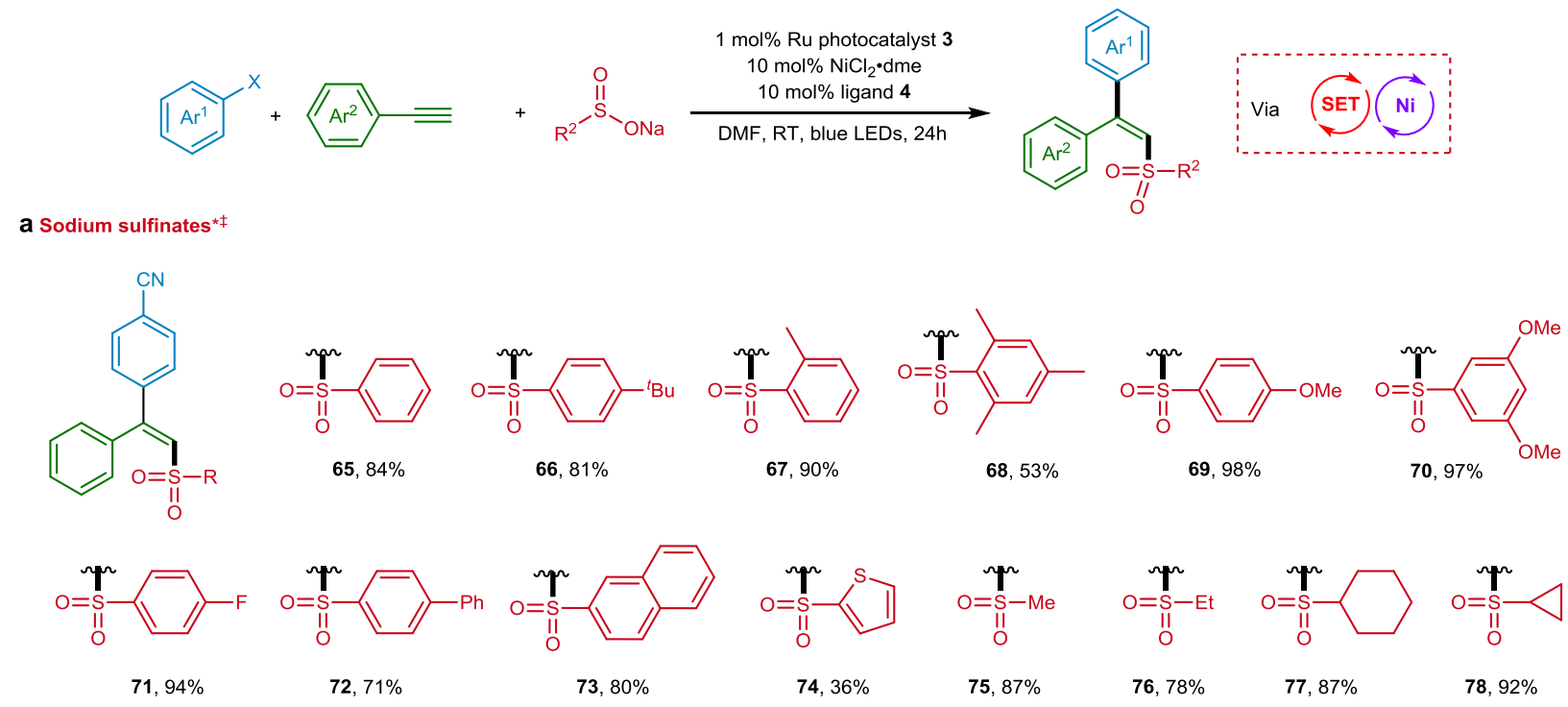

b Complex molecules<smiles>[3H]C=C(c1ccccc1)c1ccc(C(=O)OC[C@H]2OC3OC(C)(C)O[C@@H]3[C@H]2OC(C)(C)C)cc1</smiles>

79, $86 \%$ *

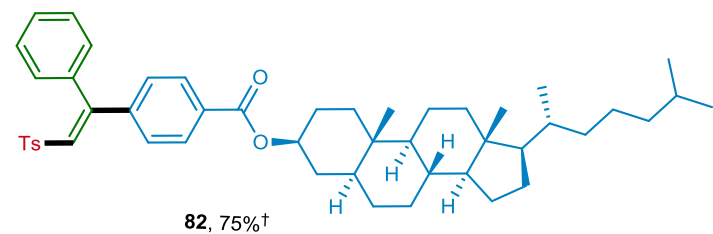

82, $75 \%{ }^{\dagger}$

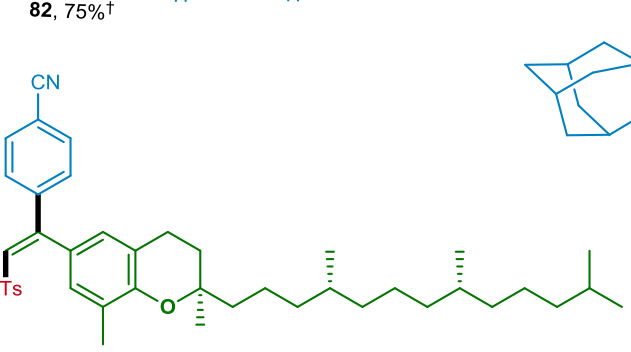

$85,67 \% *$

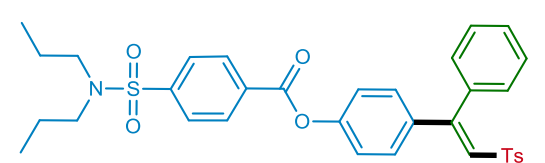

$80,67 \%$ †

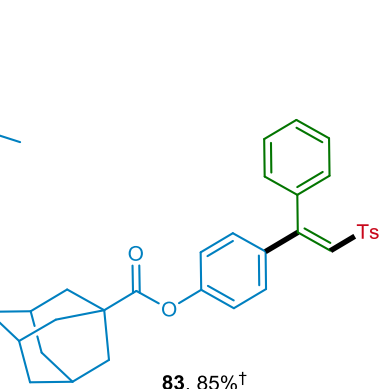

$83,85 \%^{\dagger}$

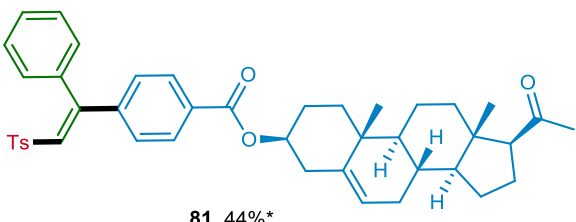

81, $44 \%$ *
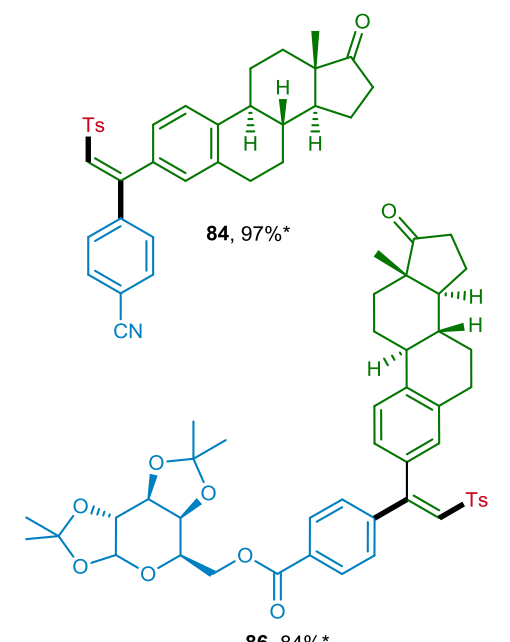

$86,84 \%$ *

Figure 4 | Substrate scope of sodium sulfinates and complex molecules for the photoredox/nickel dual catalysed aryl-sulfonylation of alkynes using Ru photocatalyst 3. Reaction conditions: Aryl halide $(0.4 \mathrm{mmol})$, alkyne $(0.4 \mathrm{mmol})$, sodium sulfinate $(0.2 \mathrm{mmol})$, Ru photocatalyst $\mathbf{3}(0.002 \mathrm{mmol})$, NiCl $2 \cdot \mathrm{dme}(0.02$ $\mathrm{mmol})$, ligand $4(0.02 \mathrm{mmol})$, degassed DMF $(2 \mathrm{~mL})$, room temperature, irradiation with $7.4 \mathrm{~W}$ blue LED strips with fan cooling for $24 \mathrm{~h}$. Yield after purification. The reactions of complex molecules $\mathbf{8 1}, \mathbf{8 2}, \mathbf{8 4 - 8 6}$ were conducted on $0.1 \mathrm{mmol}$. *From aryl bromide $\uparrow$ From aryl iodide $₫$ Aryl halide as limiting reagent $(0.2 \mathrm{mmol})$, alkyne $(0.4 \mathrm{mmol})$, sodium sulfinate $(0.4 \mathrm{mmol})$. $§$ The $Z / E$ ratio of product $\mathbf{8 0}$ is 22:78.

Next, the substrate scope was evaluated with respect to the three components in the presence of Ir-based photocatalyst 2 which, in contrast to Ru-based photocatalyst 3, promotes a reaction pathway involving SET, nickel catalysis, and energy transfer (Fig. 5). Aryl halides bearing electron-withdrawing groups and heterocyclic aryl halides gave mainly the corresponding isomerised products 
87-96 in high yield with efficient stereoselectivity. In contrast, aryl halides possessing electron-donating groups such as methyl, tert-butyl, and methoxy failed to undergo isomerisation, giving the corresponding anti-addition products 18-20. Alkynes bearing both electron-donating and electron-withdrawing groups on the aromatic rings and 3-ethynylthiophene led to efficient isomerisation of the desired products (97-101). Similarly, sodium aryl sulfinates bearing methoxy and fluoride groups and sodium alkyl sulfinates also gave the corresponding products 103-109 in good yields with good isomeric ratios. Notably, the isomerisation occurred with reasonable stereoselectivity when complex molecules were exploited (108-111), even when two complex precursors were used in the reaction (109).
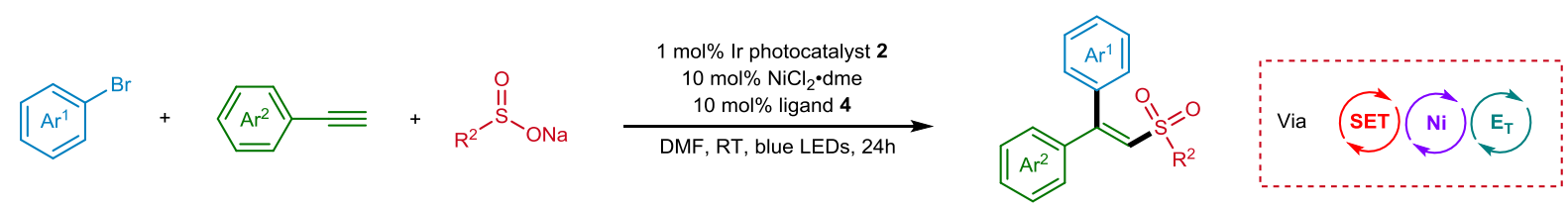<smiles>[134I]=Cc1ccccc1</smiles>

b Alkynes<smiles>N#Cc1ccc(C(=C[18F])c2ccccc2)cc1</smiles><smiles>CC=CC=CC=C(C)C</smiles>

97, $92 \%(83: 17)$
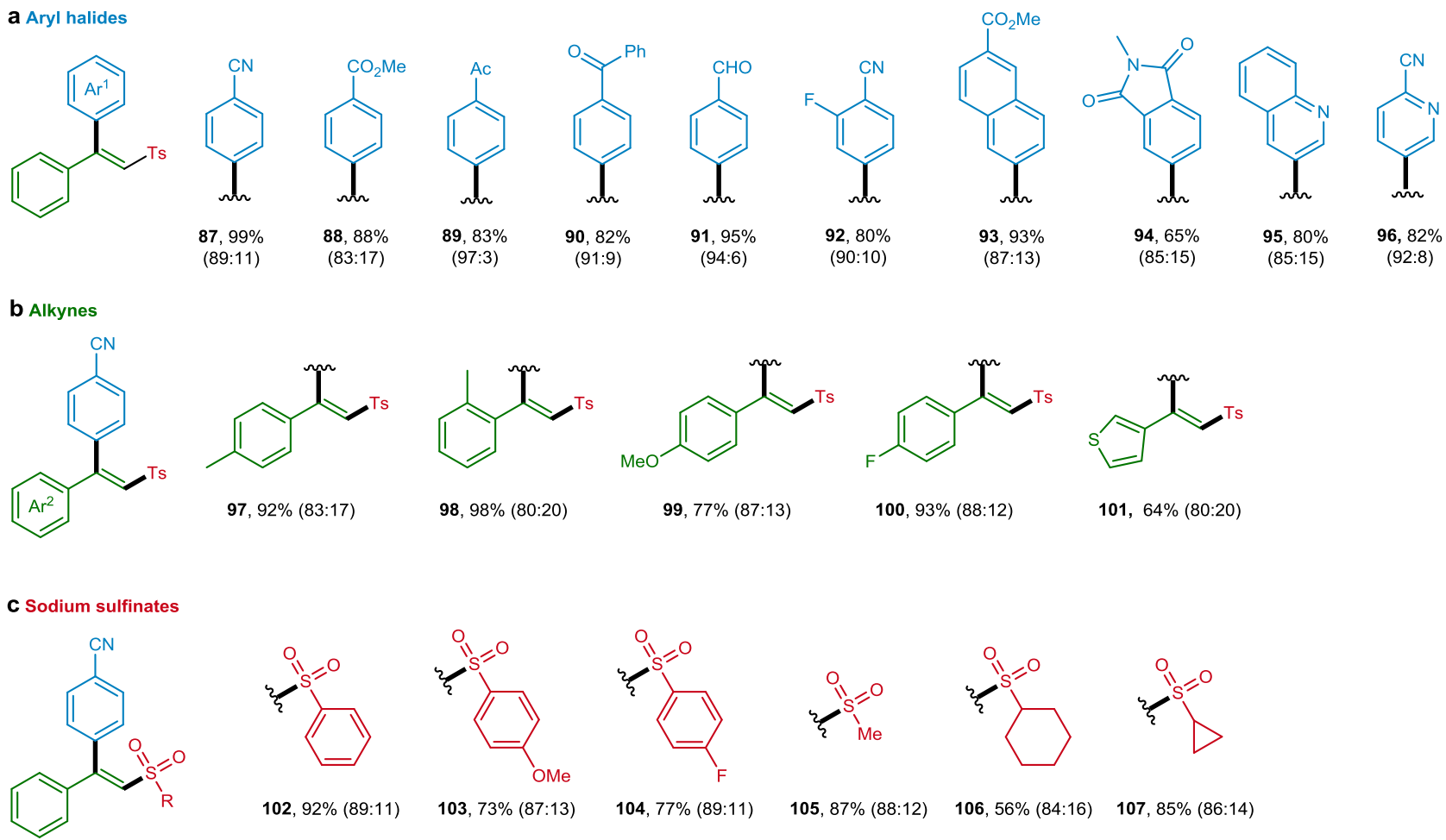

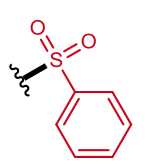

$102,92 \%(89: 11)$

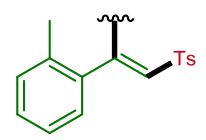

98, $98 \%(80: 20)$<smiles>CC=C(C)c1ccc(OC)cc1</smiles>

99, $77 \%(87: 13)$

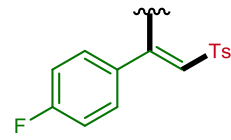

$100,93 \%(88: 12)$

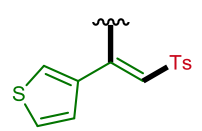

101, $64 \%(80: 20)$
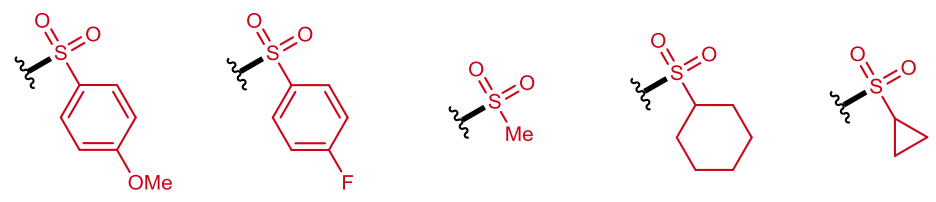

$103,73 \%(87: 13)$

104, $77 \%(89: 11)$

$105,87 \%(88: 12)$

$106,56 \%(84: 16) \quad 107,85 \%(86: 14)$

d Complex molecules

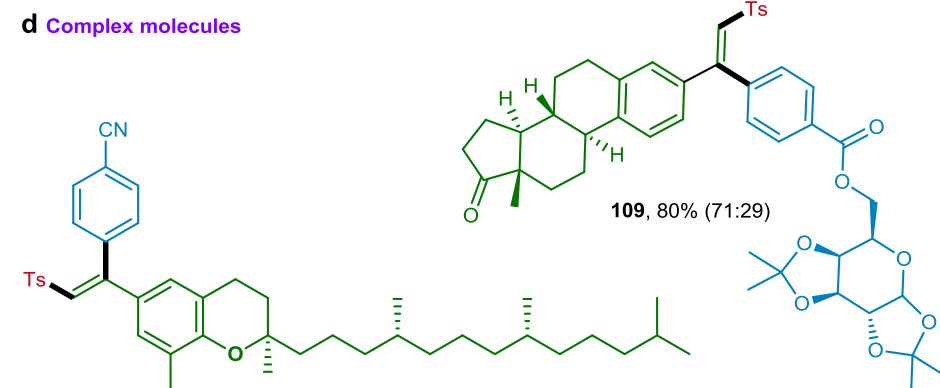

$108,52 \%(78: 22)$

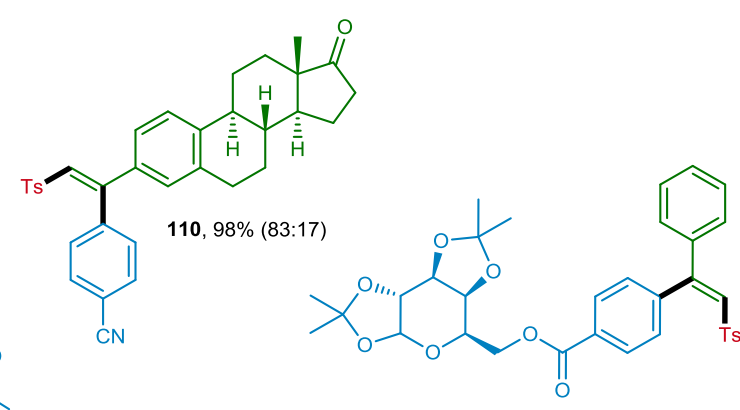

$111,90 \%(79: 21)$

Figure $\mathbf{5}$ | Substrate Scope for the photoredox/nickel dual catalysed aryl-sulfonylation of alkynes using Ir photocatalyst 2. Reaction conditions: Aryl bromides $(0.4 \mathrm{mmol})$, alkynes $(0.4 \mathrm{mmol})$, sodium sulfinates $(0.2 \mathrm{mmol})$, Ir photocatalyst $2(0.002 \mathrm{mmol}), \mathrm{NiCl}_{2} \cdot \mathrm{dme}(0.02 \mathrm{mmol})$, ligand 4 (0.02 mmol), degassed DMF (2 $\mathrm{mL}$ ), room temperature, irradiation with $34 \mathrm{~W}$ blue LED with fan cooling for $24 \mathrm{~h}$. Yield after purification. The values in parenthesis represent the ratio of the two isomers $\left(\mathbf{P}_{\text {syn }}: \mathbf{P}_{\text {anti }}\right)$ which was determined by ${ }^{1} \mathrm{H}$ NMR analysis. The reaction of complex molecules 108-110 were conducted on a $0.1 \mathrm{mmol}$ scale.

Applications of this protocol. Importantly, gram-scale reactions were successfully conducted using the standard substrate 4-bromobenzonitrile, giving both $E$ and $Z$ products in $92 \%$ and $96 \%$ yield, respectively. Although the catalytic process using Ir 2 gave the product with 91:9 Z/E stereoselectivity, the pure $Z$ isomer could be obtained in good yield after one simple recrystallisation of the product. Moreover, further functionalisation of the generated pure $E$ and $Z$ products were conducted via the cross-coupling reaction with the activated primary amine (pyridinium salt) under irradiation with blue LEDs in the absence of photocatalyst ${ }^{30}$, 
affording the corresponding products 112 and 113 in good yield with good stereoselectivity. Also, the reaction of product 114 with 1-phenylpyrrolidine and $N$-Boc proline allowed alkylation of the alkene (115 and 116) (Fig. 6a) ${ }^{31}$. Notably, this newly developed multicomponent methodology could also be easily extended to other radical precursor such as feedstock carboxylic acid. With minor reaction condition modifications, both cyclic and acyclic $N$-Boc-protected amino acid could give the desired three-component products 117-119 in good yields with good stereoselectivity (Fig. 6b). These applications demonstrate the great potential synthetic value of this visible light mediated protocol.
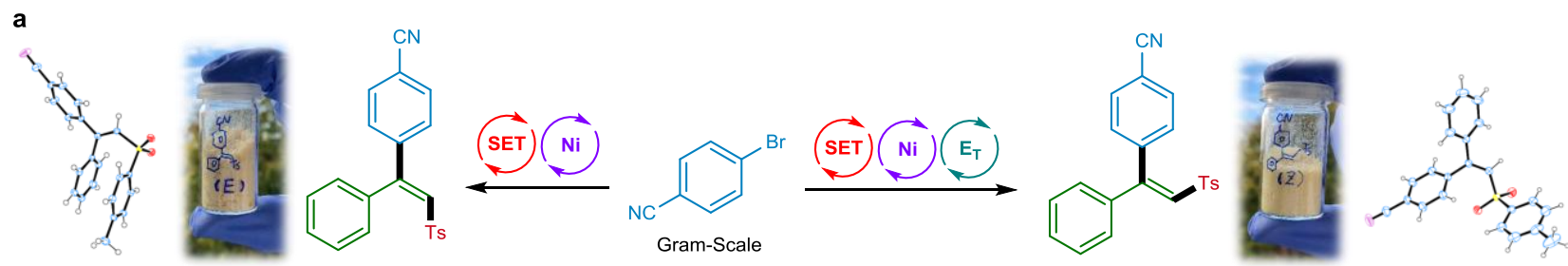

5, $92 \%(1.65 \mathrm{~g})$, pure $E$
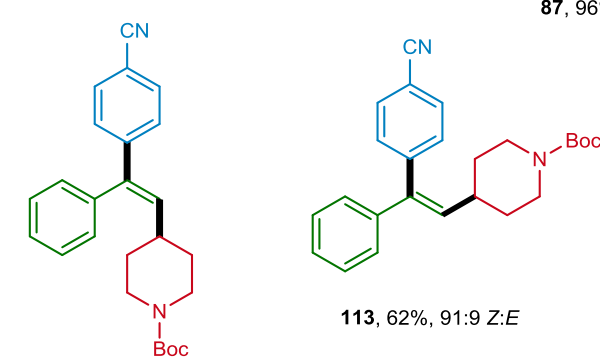

after recrystallisation:
$74 \%(1.33 \mathrm{~g})$, pure $Z$

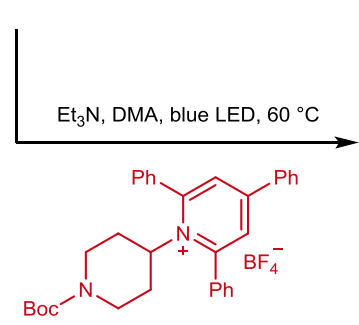

112, 77\%, 9:91 Z:E

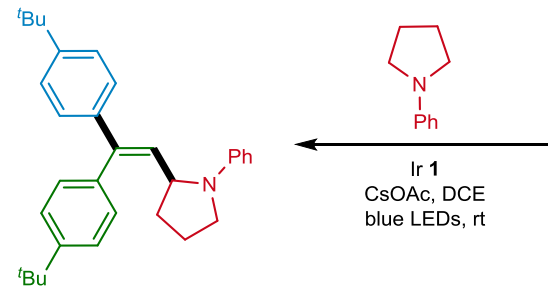

$115,88 \%$

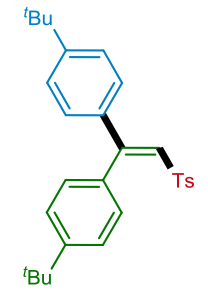

114, $87 \%$

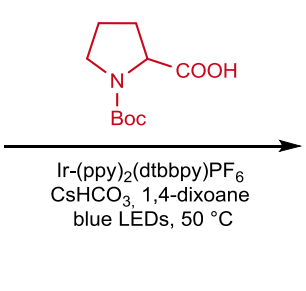

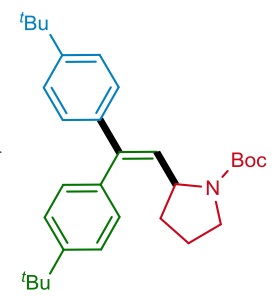

$116,82 \%$ b

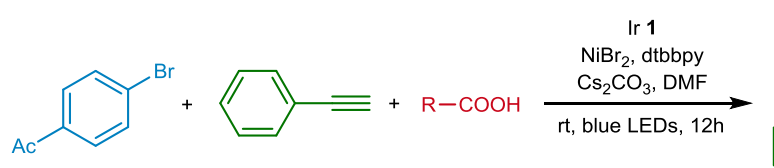

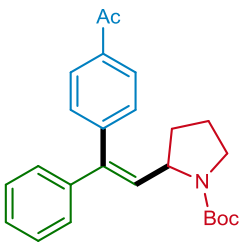

117, 66\%, 85:15 Z:E

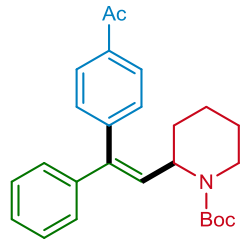

118, 74\%, 78:22 Z:E

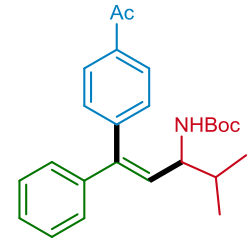

119, 73\%, 80:20 Z:E

Figure 6 | Gram-scale reactions and Applications. a, Gram-scale reactions and further transformations of the generated products. b, The utilisation of another type of radical precursor.

Mechanistic study. To gain some insight into the mechanism, we conducted a series of control experiments. Stern-Volmer quenching experiments of all three starting materials show that only sodium 4-methylbenzenesulfinate quenches the photocatalyst $\mathbf{2}$, suggesting that the process involves sulfonyl radical intermediates (Fig. 7a). Monitoring the reaction progress and representing the yield and $Z / E$ ratio $v s$ reaction time shows that $E$ isomer $\mathbf{5}$ was obtained prior to $Z$ isomer $\mathbf{8 7}$ which indicates the SET process is faster than ET process (Fig. 7b). The emission spectra of the three photocatalysts were measured, and, as shown in Fig. $7 \mathrm{c}, \lambda_{\max }(\mathbf{I r} \mathbf{1})=$ $481.6 \mathrm{~nm}, \lambda_{\max }(\operatorname{Ir} 2)=563.7 \mathrm{~nm}, \lambda_{\max }(\mathbf{R u} 3)=609.3 \mathrm{~nm}$, which means that the triplet state energy has the following trend: $\mathbf{I r} \mathbf{1}>\mathbf{I r}$ $2>\operatorname{Ru} 3\left(\mathrm{E}_{\mathrm{T}}\right.$ of $59.4,50.7$ and $46.9 \mathrm{kcal} / \mathrm{mol}$, respectively). In addition, we observed efficient isomerisation for pure $E-5$ product in the presence of Ir 2, almost no isomerisation for pure Z-87 product in the presence of $\mathbf{R u} \mathbf{3}$, and inefficient isomerisation for pure Z-87 product in the presence of $\mathbf{I r} \mathbf{1}$, indicating the role of triplet-triplet energy transfer for the isomerisation (Fig. 7d). 

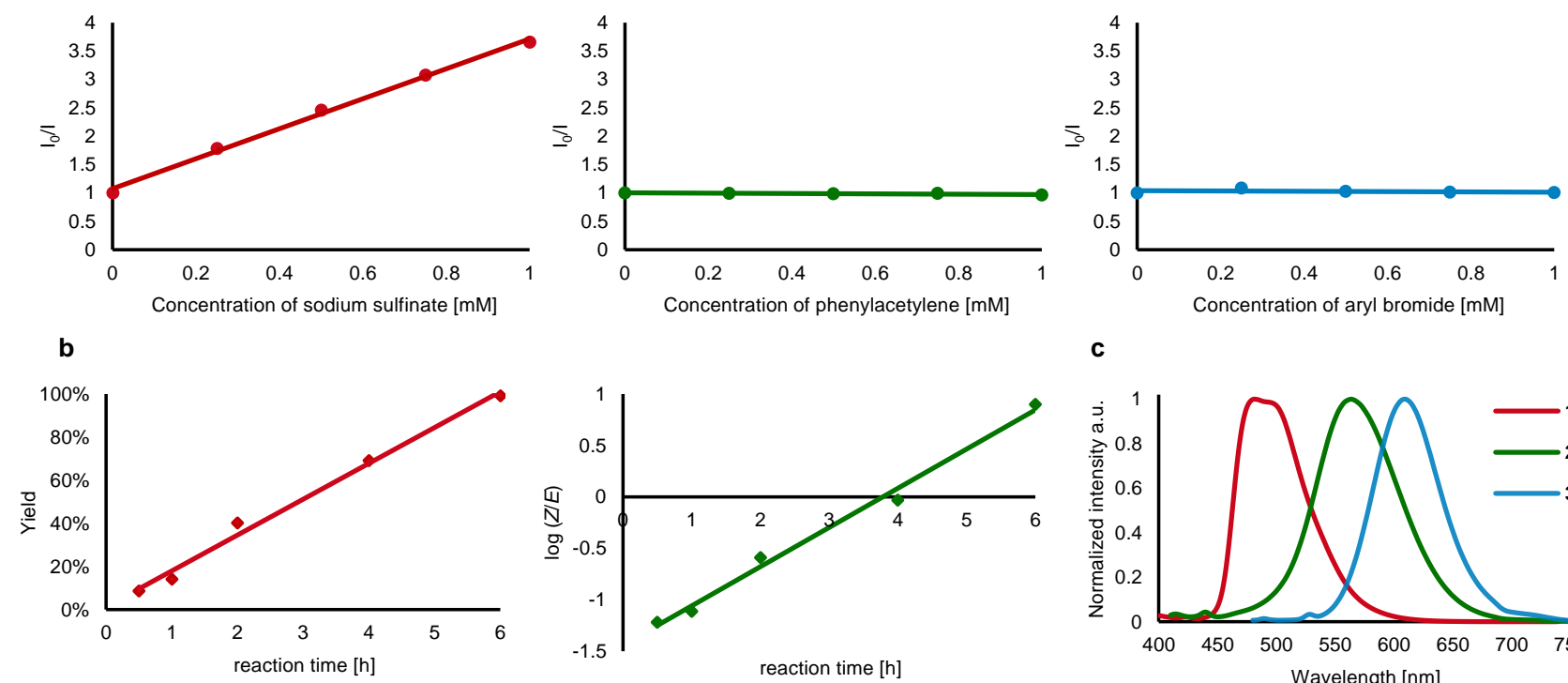

c

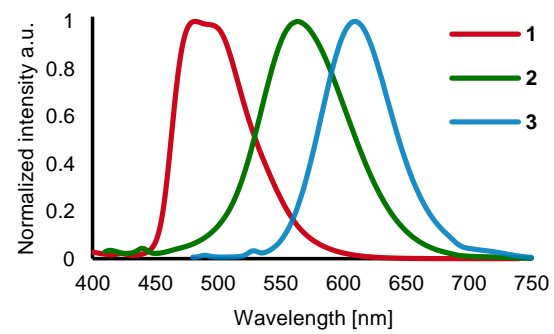

d

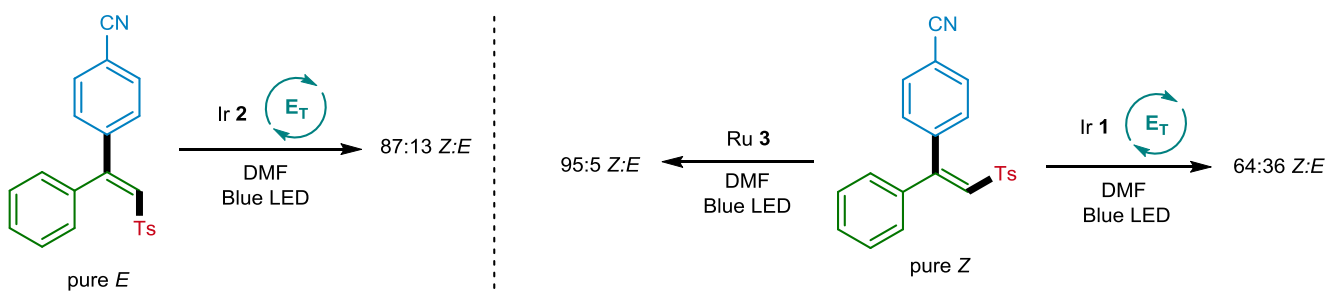

Figure $7 \mid$ Mechanistic investigations. a, Stern-Volmer quenching experiments of the starting materials. b, Reaction progress monitoring the yield and Z/E ratio $v s$ reaction time. c, Emission spectra measurement of photocatalysts. d, Z/E isomerisation via energy transfer using photocatalysts possessing different triplet state energies.

To further support the proposed mechanism, we examined the nickel catalytic cycle in detail, using DFT based calculations. The energy profile is displayed in Fig. 8a and the structures of the transition states are shown in Fig. 8b. It is well known that dual photoredox/Ni-catalysed processes are terminated by the reductive elimination from $\mathrm{Ni}^{\mathrm{III}}$ with the formation of common intermediate $\mathrm{Ni}^{\mathrm{I}}-\mathrm{Br}^{32}$. Therefore, $\mathrm{Ni}^{\mathrm{I}}-\mathrm{Br} \mathbf{E}$ was assumed as starting complex for the investigation of the nickel catalytic cycle. The catalytic process is initiated by the barrierless addition of the sulfonyl radical to $\mathrm{Ni}^{\mathrm{I}}$, leading to intermediate $\mathbf{F}$ with a free energy gain of $22.0 \mathrm{kcal} / \mathrm{mol}$ (Fig. 8) $)^{33,34}$. As alternatives, we explored the reduction of $\mathrm{Ni}^{\mathrm{I}}$ to $\mathrm{Ni}^{0}$ via SET by $\operatorname{Ir}^{\mathrm{II}} \boldsymbol{2}$ (Supplementary Fig. 13), and the addition of the sulfonyl radical to the alkyne with formation of a vinyl radical (Supplementary Fig. 14), but we found that these routes are clearly disfavoured. Next, irreversible SET from $\operatorname{Ir}^{\mathrm{II}} \mathbf{2}$ to $\mathbf{F}$ leads to the formation of the $\mathrm{Ni}^{\mathrm{I}}$ intermediate $\mathbf{G}$, a step exergonic by $17.9 \mathrm{kcal} / \mathrm{mol}$. The next section in the cycle is coordination and migratory insertion of the alkyne. It starts with the almost thermoneutral coordination of the alkyne to $\mathbf{G}$, leading to $\mathbf{H}$, followed by rearrangement of the sulfinate from a Ni-S to a Ni-O bonding mode, leading to the similarly stable intermediate I with a relatively low energy barrier of only $7.8 \mathrm{kcal} / \mathrm{mol}$. The sulfonyl group of $\mathbf{I}$ is in a suitable orientation to promote nucleophilic attack to the $\mathrm{C}^{\alpha}$ atom of the alkyne through the sulfur atom. This step occurs via the five membered cyclic transition state I-J and it has an activation barrier of $19.3 \mathrm{kcal} / \mathrm{mol}$. The resulting vinyl intermediate $\mathbf{J}$, presenting an anti orientation of the sulfonyl and phenyl substituents, is $4.3 \mathrm{kcal} / \mathrm{mol}$ lower in energy than $\mathbf{I}$. Migratory insertion of the sulfonyl group to the $C^{\beta}$ atom of the alkyne was found to be disfavoured by $2.5 \mathrm{kcal} / \mathrm{mol}$ (Supplementary Fig. 18).

At this stage, intermediate $\mathbf{J}$ can either undergo direct oxidative addition of the aryl-bromide via transition state $\mathbf{J}-\mathbf{L}$, with an activation energy of $26.6 \mathrm{kcal} / \mathrm{mol}$, leading to the final product $\mathbf{P}_{\text {syn }}$ (red pathway in Fig. 8a), or it can undergo a two step route (blue pathway in Fig. 8a), starting with the anti to syn isomerisation of the vinyl group, $\mathbf{J}$ to $\mathbf{K}$, followed by oxidative addition of the aryl-bromide, $\mathbf{K}$ to $\mathbf{L}$, leading to the final product $\mathbf{P}_{\text {anti }}$. The anti to syn isomerisation occurs via transition state $\mathbf{J}-\mathbf{K}$, with an energy barrier of $23.3 \mathrm{kcal} / \mathrm{mol}$, and it involves a rotation around the $\mathrm{C}-\mathrm{C}$ bond. The relatively low energy barrier for this rotation is due to 
delocalisation of the $\pi$-system of the vinyl bond to include the Ni atom in transition state $\mathbf{J}-\mathbf{K}$, which reduces the double bond character of the $\mathrm{C}=\mathrm{C}$ bond (see Supplementary Table 7). The oxidative addition of the aryl-bromide occurs via transition state $\mathbf{K}-\mathbf{L}$, laying $1.9 \mathrm{kcal} / \mathrm{mol}$ below transition state $\mathbf{J}-\mathbf{K}$. Comparison of the blue and red routes in Fig. 8a indicates that the two step route is favoured, as transition state $\mathbf{J}-\mathbf{K}$ is favoured by $3.3 \mathrm{kcal} / \mathrm{mol}$ over transition state $\mathbf{J}-\mathbf{L}$ ', which implies that intermediate $\mathbf{J}$ preferentially evolves to intermediate $\mathbf{L}$. This result is consistent with the experimental selective formation of $\mathbf{P}_{\text {anti }}$ (Fig. $7 \mathrm{~d}$ ).

Release of the product via reductive elimination from $\mathbf{L}$ requires isomerisation of the system to the more stable intermediate $\mathbf{N}$, which presents a cis disposition of the aryl and vinyl groups. The lowest pathway we found involves $\mathrm{Br}^{-}$dissociation/coordination via intermediate $\mathbf{M}$. The reductive elimination of product $\mathbf{P}_{\text {anti }}$ from $\mathbf{N}$ occurs via transition state $\mathbf{N}$-E and a low energy barrier of only $5.0 \mathrm{kcal} / \mathrm{mol}$.

a

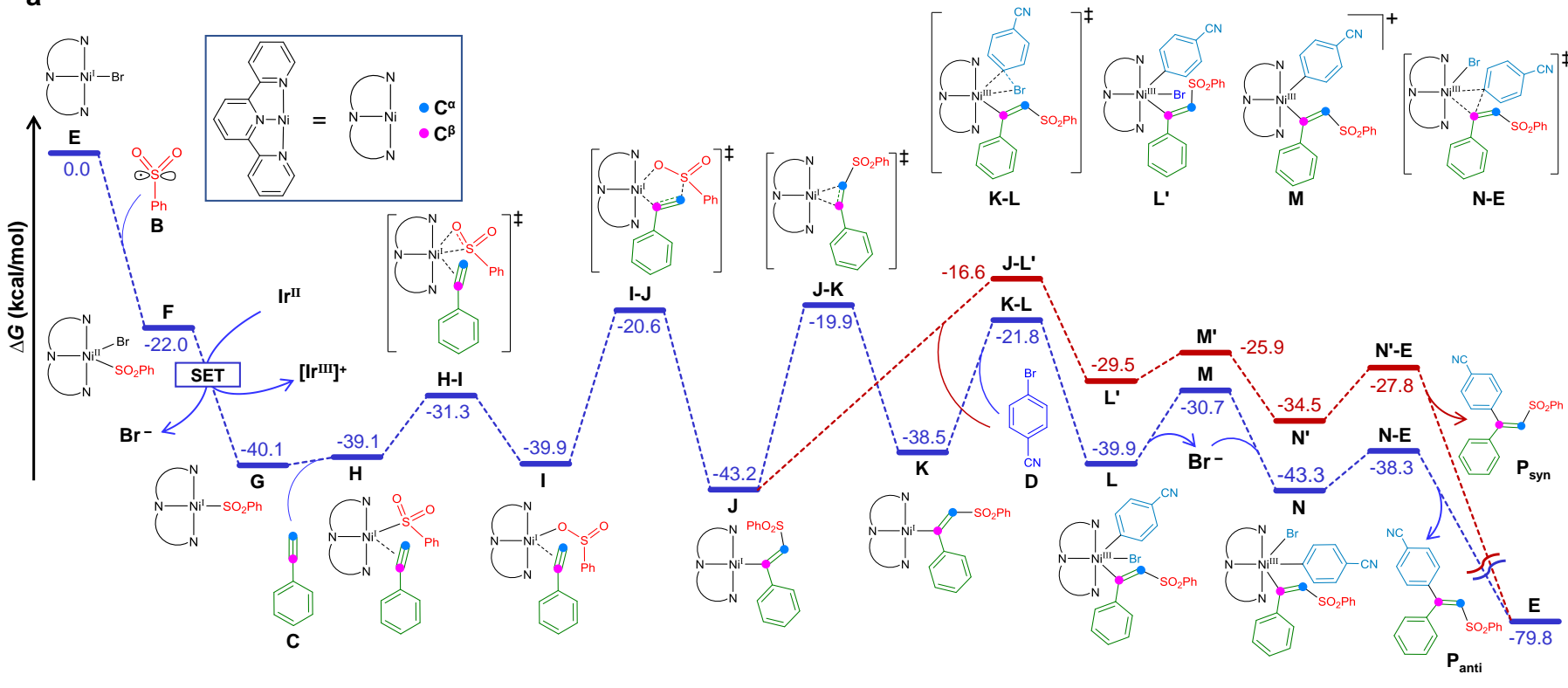
$\leftarrow \underset{\text { addition }}{\text { radical }} \rightarrow \leftarrow$ SET $\rightarrow \longleftarrow$ migratory insertion of alkyne

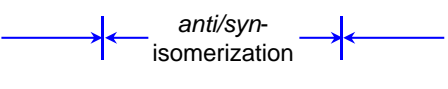
oxidative addition

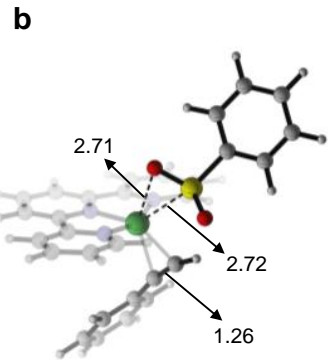

H-I

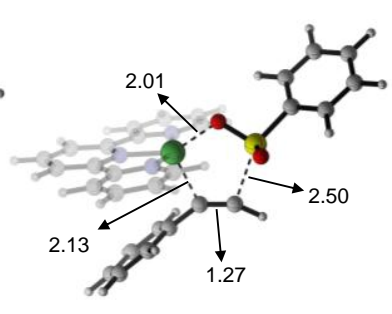

I-J

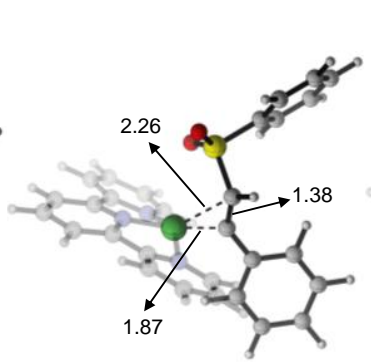

J-K
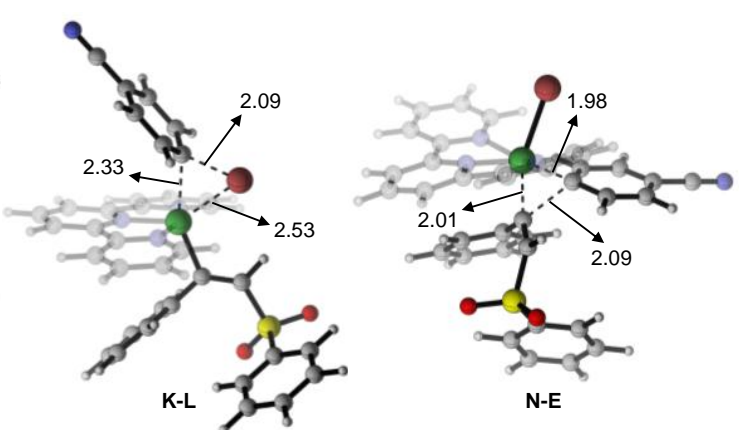

Figure 8 Computational investigation of the nickel catalytic cycle. a, Proposed mechanism of nickel catalytic cycle based on DFT calculations. b, DFT optimised geometries of all transition states involved in the lowest energy pathway. Bond lengths are in $\AA$.

\section{Conclusion}

In conclusion, we have developed a three-component cross coupling reaction involving aryl halides, sodium sulfinates and alkynes. The protocol exhibits high tolerance towards various functional groups and could be readily extended to diverse complex molecules. The proposed mechanism features the combination of single electron transfer (SET) with nickel catalysis and single electron transfer (SET), nickel catalysis followed by energy transfer (ET), enabling the generation of trisubstituted alkene isomers. We anticipate that this protocol will provide a platform for other mild multi-component cross coupling reactions in organic synthesis.

\section{Methods}

General procedure for the catalytic reactions using Ru 3 as photocatalyst. A dry reaction tube equipped with a Teflon-coated magnetic stir bar was charged with aryl 
halide $\left(0.4 \mathrm{mmol}, 2\right.$ equiv., if solid), sodium sulfinate $\left(0.2 \mathrm{mmol}, 1\right.$ equiv.), Ru $3(2.2 \mathrm{mg}, 0.002 \mathrm{mmol}, 1 \mathrm{~mol} \%), \mathrm{NiCl}_{2} \cdot \mathrm{dme}(4.4 \mathrm{mg}, 0.02 \mathrm{mmol}, 10 \mathrm{~mol} \%)$, 4,4',4"-tri-tert-butyl-2,2':6',2"-terpyridine ( $8.0 \mathrm{mg}, 0.02 \mathrm{mmol}, 10 \mathrm{~mol} \%)$ and alkyne ( $0.4 \mathrm{mmol}, 2$ equiv., if solid). It was capped with a rubber septum, evacuated and backfilled with argon (3 times). Then, degassed DMF ( $2.0 \mathrm{~mL})$, aryl halide ( $0.4 \mathrm{mmol}, 2$ equiv., if liquid) and alkyne ( $0.4 \mathrm{mmol}, 2$ equiv., if liquid) were added via syringe. The reaction mixture was stirred at room temperature for $24 \mathrm{~h}$ under irradiation with $7.4 \mathrm{~W}$ blue LED strips with fan cooling. Upon completion, the solution was diluted with $\mathrm{H}_{2} \mathrm{O}(10 \mathrm{~mL})$ and EtOAc $(10 \mathrm{~mL})$. The layers were separated, then the aqueous layer was extracted with EtOAc. The combined organic layer was washed with $\mathrm{H}_{2} \mathrm{O}(20 \mathrm{~mL})$ and brine $(20 \mathrm{~mL})$. The organic layer was dried with anhydrous $\mathrm{Na}_{2} \mathrm{SO}_{4}$, then concentrated under vacuum and purified by column chromatography on silica gel using hexane: EtOAc as eluent to get the corresponding pure product (The reaction of complex molecules $\mathbf{8 1}, \mathbf{8 2}, \mathbf{8 4 - 8 6}$ were conducted on $0.1 \mathrm{mmol}$ with $2 \mathrm{~mL}$ DMF solvent).

General procedure for the catalytic reactions using Ir 2 as photocatalyst. A dry reaction tube equipped with a Teflon-coated magnetic stir bar was charged with aryl halide ( $0.4 \mathrm{mmol}, 2$ equiv., if solid), sodium sulfinate $\left(0.2 \mathrm{mmol}, 1\right.$ equiv.), Ir $2(2.1 \mathrm{mg}, 0.002 \mathrm{mmol}, 1 \mathrm{~mol} \%), \mathrm{NiCl}_{2} \cdot \mathrm{dme}(4.4 \mathrm{mg}, 0.02 \mathrm{mmol}, 10 \mathrm{~mol} \%)$, 4,4',4"-tri-tert-butyl-2,2':6',2"-terpyridine ( $8.0 \mathrm{mg}, 0.02 \mathrm{mmol}, 10 \mathrm{~mol} \%)$ and alkyne ( $0.4 \mathrm{mmol}, 2$ equiv., if solid). It was capped with a rubber septum, evacuated and backfilled with argon ( 3 times). Then, degassed DMF ( $2.0 \mathrm{~mL})$, aryl halide $(0.4 \mathrm{mmol}, 2$ equiv., if liquid) and alkyne ( $0.4 \mathrm{mmol}, 2$ equiv., if liquid) were added via syringe. The reaction mixture was stirred at room temperature for $24 \mathrm{~h}$ under irradiation with $34 \mathrm{~W}$ Kessil blue LED lamp with fan cooling. Upon completion, the solution was diluted with $\mathrm{H}_{2} \mathrm{O}(10 \mathrm{~mL})$ and EtOAc $(10 \mathrm{~mL})$. The layers were separated, then the aqueous layer was extracted with EtOAc. The combined organic layer was washed with $\mathrm{H}_{2} \mathrm{O}(20$ $\mathrm{mL}$ ) and brine $(20 \mathrm{~mL})$. The organic layer was dried with anhydrous $\mathrm{Na}_{2} \mathrm{SO}_{4}$, then concentrated under vacuum and purified by column chromatography on silica gel using hexane: EtOAc as eluent to get the corresponding pure product (The reaction of complex molecules 108-110 were conducted on $0.1 \mathrm{mmol}$ with $2 \mathrm{~mL}$ DMF solvent).

Computational details. All DFT-calculations were performed using Gaussian 09, Revision D. $01^{35}$. The geometry optimisation and frequency analysis were performed using the PBE functional ${ }^{36,37}$. The split-valence plus one polarisation function def2-SVP basis set was used for non-metal atoms and the triple- $\zeta$ basis set def2-TZVP was used for metals $^{38,39}$. In all cases, the default integral grid (Fine Grid) was employed. Frequency calculations were performed in order to obtain thermal corrections (298 K) and to confirm the nature of the stationary points (minima with no imaginary frequency or transition states with one imaginary frequency). For transition state structures, IRC calculations were undertaken to confirm that the transition states were connected to the correct minima. For better energetics, single point energy calculations were performed using the hybrid-meta-GGA M06 functional ${ }^{40}$ with the def2-TZVPP basis set ${ }^{38,39}$ for all atoms. Restricted DFT was employed for species in the singlet spin state, while unrestricted DFT was used for species in the doublet or triplet spin states. The implicit SMD solvent model ${ }^{41}$ was used to account for solvent effects, $N, N$-Dimethylformamide (DMF, $\varepsilon=37.2$ ). Unless specified otherwise, Gibbs free energy, $G$, was used throughout the text. The $G$ value was obtained by augmenting the in solvent electronic energy $E_{\mathrm{el}}$ at the M06(SMD-DMF)/(def2TZVPP) level with the respective free energy corrections at the PBE/def2-SVP(C,H,N,O,S,Br)/def2-TZVP(Ni,Ir) level in gas phase. As it is known that translational and rotational entropies in solution for association/dissociation processes are overestimated/underestimated, and that the deviation in the free energies is approximately $1.89 \mathrm{kcal} / \mathrm{mol}$ from the standard state $(1 \mathrm{~atm})$ to $1 \mathrm{M}$ in solution ${ }^{42,43}$, we have reduced by $1.89 \mathrm{kcal} / \mathrm{mol}$ the free energy for addition steps and we have added $1.89 \mathrm{kcal} / \mathrm{mol}$ to the free energy of the dissociation steps. Natural bond orbital (NBO) analysis were performed using the $N B O 3.1$ $\operatorname{program}^{44,45}$. The geometries were realised using CYLview, $1.0 \mathrm{~b}^{46}$. The Mayer bond order was calculated using the Multiwfn 3.5 software ${ }^{47}$.

\section{Data availability}

The X-ray crystallographic coordinates for structures of $\mathbf{5}$ and $\mathbf{8 7}$ reported in this Article have been deposited at the Cambridge Crystallographic Data Centre (CCDC) under deposition numbers CCDC 1914140 (5) and 1914141 (87). These data can be obtained free of charge from The Cambridge Crystallographic Data Centre via www.ccdc.cam.ac.uk/data_request/cif. Experimental procedures and characterisation of the new compounds are available in the Supplementary Information. All other data are available from the authors upon reasonable request.

\section{References}

1. Tietze, L. F. \& Modi, A. Multicomponent domino reactions for the synthesis of biologically active natural products and drugs. Med. Res. Rev. 20, 304-322 (2000).

2. Masson, G., Neuville, L., Bughin, C., Fayol, A. \& Zhu, J. Multicomponent syntheses of macrocycles. Top. Heterocycl. Chem. 25, 1-24 (2000).

3. Domling, A., Wang, W. \& Wang, K. Chemistry and Biology of Multicomponent Reactions. Chem. Rev. 112, 3083-3135 (2012).

4. Levi, L. \& Müller, T. J. J. Multicomponent syntheses of functional chromophores. Chem. Soc. Rev. 45, 2825-2846 (2016).

5. Zhu, J., Wang, Q. \& Wang, M.-X. Eds. Multicomponent Reactions in Organic Synthesis. (Wiley-VCH, 2015).

6. Müller, T. J. J. \& Deilhof, K. Alkynes in Multicomponent Synthesis of Heterocycles in Multicomponent Reactions in Organic Synthesis. Zhu, J., Wang, Q. \& Wang, M.-X. Eds. (Wiley-VCH, 2015) 333-378.

7. Xue, F., Zhao, J., Hor, T. S. A. \& Hayashi, T. Nickel-Catalyzed Three-Component Domino Reactions of Aryl Grignard Reagents, Alkynes, and Aryl Halides Producing Tetrasubstituted Alkenes. J. Am. Chem. Soc. 137, 3189-3192 (2015).

8. Satoh, T., Ogino, S., Miura, M. \& Nomura, M. Synthesis of Highly Substituted 1,3-Butadienes by Palladium-Catalyzed Arylation of Internal Alkynes. Angew. Chem. Int. Ed. 43, 5063-5065 (2004).

9. Kischkewitz, M., Okamoto, K., Mück-Lichtenfeld, C. \& Studer, A. Radical-polar crossover reactions of vinylboron ate complexes. Science 355, 936-938 (2017).

10. Lu, Q. et al. Dioxygen-Triggered Oxidative Radical Reaction: Direct Aerobic Difunctionalization of Terminal Alkynes toward $\beta$-Keto Sulfones. J. Am. Chem. Soc. 135, 11481-11484 (2013).

11. García-Domínguez, A., Müller, S. \& Nevado, C. Nickel-Catalyzed Intermolecular Carbosulfonylation of Alkynes via Sulfonyl Radicals. Angew. Chem. Int. Ed. 56, 9949-9952 (2017).

12. Li, Z., Garcia-Dominguez, A. \& Nevado, C. Nickel-Catalyzed Stereoselective Dicarbofunctionalization of Alkynes. Angew. Chem., Int. Ed. 55, 6938-6941 (2016).

13. Li, Z., Garcia-Dominguez, A. \& Nevado, C. Pd-Catalyzed Stereoselective Carboperfluoroalkylation of Alkynes. J. Am. Chem. Soc. 137, 11610-11613 (2015).

14. Derosa, J., Tran, V. T., Boulous, M. N., Chen, J. S. \& Engle, K. M. Nickel-Catalyzed $\beta$, $\gamma$-Dicarbofunctionalization of Alkenyl Carbonyl Compounds via Conjunctive 
Cross-Coupling. J. Am. Chem. Soc. 139, 10657-10660 (2017).

15. Marzo, L., Pagire, S. K., Reiser, O. \& König, B. Visible-Light Photocatalysis: Does it make a difference in Organic Synthesis? Angew. Chem. Int. Ed. 57, 10034-10072 (2018).

16. Ye, J. H. et al. Visible-Light-Driven Iron-Promoted Thiocarboxylation of Styrenes and Acrylates with CO2. Angew. Chem. Int. Ed. 56, 15416-15420 (2017).

17. Koike, T. \& Akita, M. Fine Design of Photoredox Systems for Catalytic Fluoromethylation of Carbon-Carbon Multiple Bonds. Acc. Chem. Res. 49, 1937-1945 (2016).

18. Metternich, J. B. \& Gilmour, R. A Bio-Inspired, Catalytic $E \rightarrow Z$ Isomerization of Activated Olefins. J. Am. Chem. Soc. 137, 11254-11257 (2015).

19. Zuo, Z. et al. Merging photoredox with nickel catalysis: Coupling of alpha-carboxyl sp(3)-carbons with aryl halides. Science 345, 437-440 (2014).

20. Tellis, J. C., Primer, D. N. \& Molander, G. A. Single-electron transmetalation in organoboron cross-coupling by photoredox/nickel dual catalysis. Science 345, 433-436 (2014).

21. Twilton, J., Zhang, P., Shaw, M. H., Evans, R. W. \& MacMillan, D. W. C. The merger of transition metal and photocatalysis. Nat. Rev. Chem. 1, 0052 (2017).

22. Matsui, J. K., Lang, S. B., Heitz, D. R. \& Molander, G. A. Photoredox-Mediated Routes to Radicals: The Value of Catalytic Radical Generation in Synthetic Methods Development. ACS Catal 7, 2563-2575 (2017).

23. Wang, H., Lu, Q., Chiang, C. W., Luo, Y., Zhou, J., Wang, G. \& Lei, A. Markovnikov-Selective Radical Addition of S-Nucleophiles to Terminal Alkynes through a Photoredox Process. Angew. Chem. Int. Ed. 56, 595-599 (2017).

24. Yue, H., Zhu, C. \& Rueping, M. Cross-Coupling of Sodium Sulfinates with Aryl, Heteroaryl and Vinyl Halides by Nickel/photoredox dual catalysis. Angew. Chem. Int. Ed. 57, 1371-1375 (2018).

25. Deng, H.-P., Fan, X.-Z., Chen, Z.-H., Xu, Q.-H. \& Wu, J. Photoinduced Nickel-Catalyzed Chemo- and Regioselective Hydroalkylation of Internal Alkynes with Ether and Amide $\alpha$-Hetero C(sp3)-H Bonds. J. Am. Chem. Soc. 139, 13579-13584 (2017).

26. Go, S. Y., Lee, G. S. \& Hong, S. H. Highly Regioselective and E/Z-Selective Hydroalkylation of Ynone, Ynoate, and Ynamide via Photoredox Mediated Ni/Ir Dual Catalysis. Org. Lett. 20, 4691-4694 (2018).

27. Singh, K., Staig, S. J. \& Weaver, J. D. Facile Synthesis of Z-Alkenes via Uphill Catalysis. J. Am. Chem. Soc. 136, 5275-5278 (2014).

28. Fabry, D. C., Ronge, M. A. \& Magnus, R. Immobilization and Continuous Recycling of Photoredox Catalysts in Ionic Liquids for Applications in Batch Reactions and Flow Systems: Catalytic Alkene Isomerization by Using Visible Light. Chem. Eur. J. 21, 5350-5354 (2015).

29. Wei, X.-J., Boon, W., Hessel, V. \& Noël, T. Visible-Light Photocatalytic Decarboxylation of $\alpha, \beta$-Unsaturated Carboxylic Acids: Facile Access to Stereoselective Difluoromethylated Styrenes in Batch and Flow. ACS Catal 7, 7136-7140 (2017).

30. Wu, J., Grant, P. S., Li, X., Noble, A. \& Aggarwal, V. K. Catalyst-Free Deaminative Functionalizations of Primary Amines by Photoinduced Single-Electron Transfer. Angew. Chem. Int. Ed. 58, 5697-5701 (2019).

31. Noble, A. \& MacMillan, D. W. C. Photoredox $\alpha$-Vinylation of $\alpha$-Amino Acids and N-Aryl Amines. J. Am. Chem. Soc. 136, 11602-11605 (2014).

32. Gutierrez, O., Tellis, J. C., Primer, D. N., Molander, G. A. \& Kozlowski, M. C. Nickel-Catalyzed Cross-Coupling of Photoredox-Generated Radicals: Uncovering a General Manifold for Stereoconvergence in Nickel-Catalyzed Cross-Couplings. J. Am. Chem. Soc. 137, 4896-4899 (2015).

33. Oderinde, M. S. et al. Highly Chemoselective Iridium Photoredox and Nickel Catalysis for the Cross-Coupling of Primary Aryl Amines with Aryl Halides. Angew. Chem. Int. Ed. 55, 13219-13223 (2016).

34. Oderinde, M. S., Frenette, M., Robbins, D. W., Aquila, B. \& Johannes, J. W. Photoredox Mediated Nickel Catalyzed Cross-Coupling of Thiols With Aryl and Heteroaryl Iodides via Thiyl Radicals. J. Am. Chem. Soc. 138, 1760-1763 (2016).

35. Frisch, M. J. et al. Gaussian 09, Revision D.01, Gaussian, Inc. Wallingford, CT. (2013).

36. Perdew, J. P., Burke, K. \& Ernzerhof, M. Generalized Gradient Approximation Made Simple. Phys. Rev. Lett. 77, 3865-3868 (1996).

37. Perdew, J. P., Burke, K. \& Ernzerhof, M. Generalized Gradient Approximation Made Simple. Phys. Rev. Lett. 78, 1396 (1997).

38. Weigend, F. \& Ahlrichs, R. Balanced basis sets of split valence, triple zeta valence and quadruple zeta valence quality for $\mathrm{H}$ to Rn: Design and assessment of accuracy. Phys. Chem. Chem. Phys. 7, 3297-3305 (2005).

39. Weigend, F. Accurate Coulomb-fitting basis sets for H to Rn. Phys. Chem. Chem. Phys. 8, 1057-1065 (2006).

40. Zhao, Y. \& Truhlar, D. G. The M06 suite of density functionals for main group thermochemistry, thermochemical kinetics, noncovalent interactions, excited states, and transition elements: two new functionals and systematic testing of four M06-class functionals and 12 other functionals. Theor. Chem. Acc. 120, 215-241 (2008).

41. Marenich, A. V., Cramer, C. J. \& Truhlar, D. G. Universal solvation model based on solute electron density and on a continuum model of the solvent defined by the bulk dielectric constant and atomic surface tensions. J. Phys. Chem. B 113, 6378-6396 (2009).

42. Kelly, C. P., Cramer, C. J. \& Truhlar, D. G. SM6: A Density Functional Theory Continuum Solvation Model for Calculating Aqueous Solvation Free Energies of Neutrals, Ions, and Solute-Water Clusters. J. Chem. Theory Comput. 1, 1133-1152 (2005).

43. Kelly, C. P., Cramer, C. J. \& Truhlar, D. G. Aqueous Solvation Free Energies of Ions and Ion-Water Clusters Based on an Accurate Value for the Absolute Aqueous Solvation Free Energy of the Proton. J. Phys. Chem. B 110, 16066-16081 (2006).

44. Glendening, E. D., Reed, A. E., Carpenter, J. E. \& Weinhold, F. NBO Version 3.1 (1998).

45. Reed, A. E., Curtiss, L. A. \& Weinhold, F. Intermolecular interactions from a natural bond orbital, donor-acceptor viewpoint. Chem. Rev. 88, 899-926 (1988).

46. Legault, C. Y. CYLview (Unversité de Sherbrooke: Sherbrooke, QC, Canada, 1.0b, 2009) http://www.cylview.org.

47. Lu, T. \& Chen, F. Multiwfn: A multifunctional wavefunction analyser. J. Comput. Chem. 33, 580-592 (2012).

\section{Acknowledgements}

The authors thank Prof. Dr. Dominik Wöll and Oleksii Nevskyi for assistance in measuring emission spectra of photocatalysts. H.Y. thanks the China Scholarship Council. C.Z., B.M., L.C. and M.R. are acknowledge King Abdullah University of Science and Technology (KAUST) for support and the KAUST Supercomputing Laboratory for providing computational resources of the supercomputer Shaheen II. The research leading to these results has received funding from the European Research Council under the European Union's Seventh Framework Programme (FP/2007-2013)/ERC Grant Agreement no. 617044 (SunCatChem). 


\section{Author contributions}

C.Z., H.Y. and M.R. conceived and designed the experiments. C.Z., H.Y. performed and analyzed the experiments. I.A. conducted X-ray crystal structure analysis. C.Z., B.M. and L.C. performed the theoretical calculations. All authors discussed the results and contributed to the manuscript.

\section{Competing interests}

The authors declare no competing interests. 